\section{THEORELL'S PRINTING METEOROGRAPH}

SOME time since brief mention was made of the above $S$ instrument (devised by Dr. A. G. Theorell, of Nybrogatan, Stockholm, Sweden-NATURE, vol. iv. p. 466)with reference to its having been exhibited at the London International Exhibition 1871 .

Being in possession $\mathrm{f}$ a detailed description of the same, I have thought th $t$ a copy with additional remarks may be of interest in tt a pages of NATURE.

I have no hesitation. I saying that this instrument does not occupy the prominent position to which it is justly entitled, neither do? I think that it is so well knownwhether taken in the light of a wonderful piece of mechanism, or of excellent workmanship-as it well deserves to be.

The following is a description of the instrument in accordance with the original, excepting only that I have made a few slight alterations in order to render it more intelligible, the original having been, as I suppose, translated from the Swedish language, and not well expressed :-

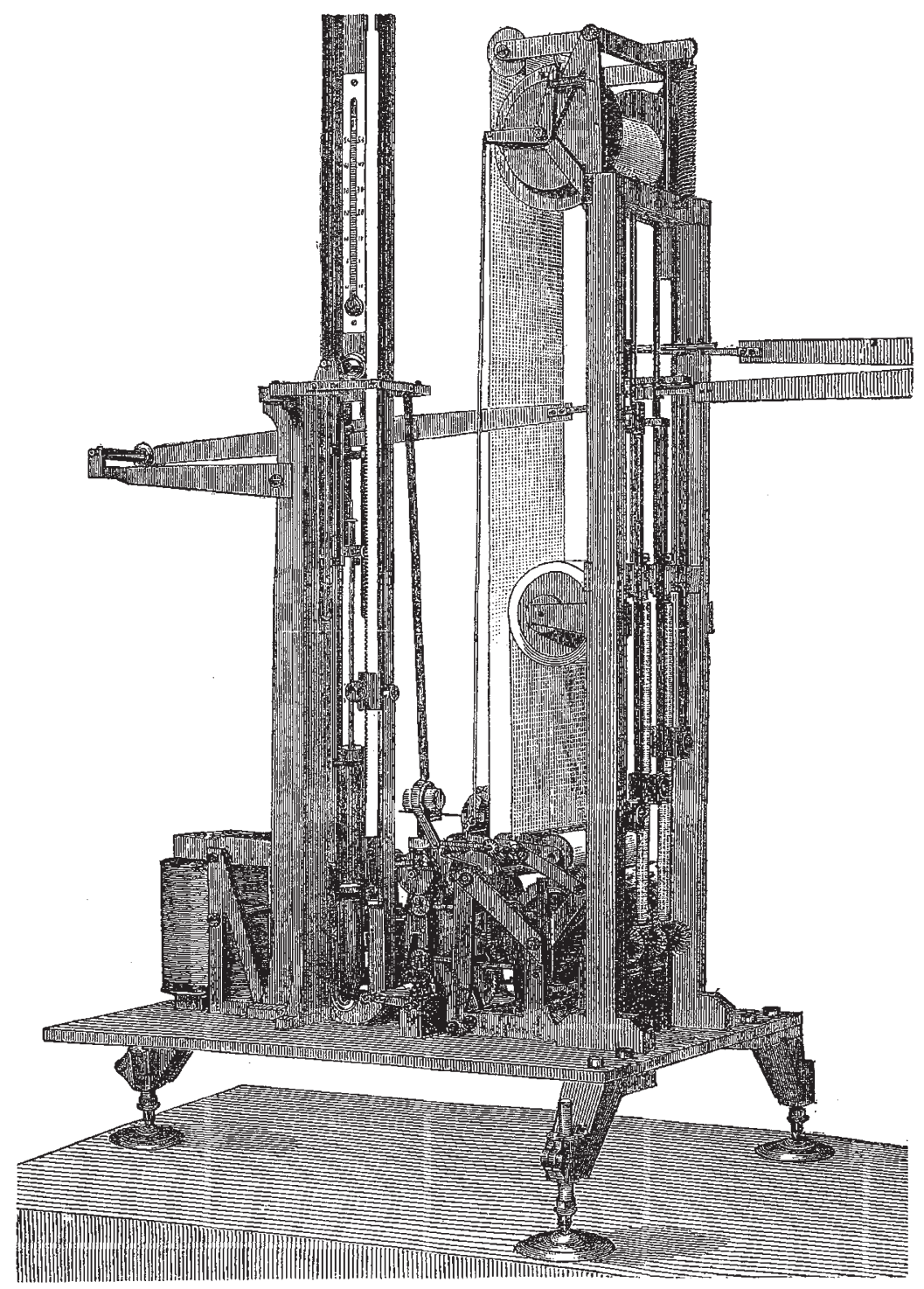

"Meteorological observations are by this instrument delivered in tables printed on a slip of paper. Of the four tabular columns the first gives the hours, the second the temperature, the third the degree of humidity according to August's method, and the fourth the atmospheric pressure; this last (atmospheric pressure) is given in millimetres, but the first figure, being always a 7 , is suppressed. The degrees of the thermometer employed are those of the centigrade scale, and negative degrees are expressed by their complements to roo.
"The registration takes place by means of electrical currents, which are closed by contact between the mercury in the various meteorological instruments and steel wires that descend into their tubes. These steel wires are connected, by means of levers and three vertical screws, each with its respective system of brass wheels with numerical type engraved on the edges, in such a manner that the rotation of the wheels causes an upward or downward motion of the steel wires, so that the point of the scale on which the lower extremity of the wire is situated, is neces- 
sarily that indicated by the number appearing at the same moment uppermost on the corresponding wheels.

"The wheels containing the figures are governed by an electro-magnetic motor, which, for "each observation, sets the three wheel systems successively in motion, until the corresponding wires have reached the mercury in their respective meteorological instruments, when the abovementioned electrical current instantly arrests the motion, so that accordingly all three steel wires stop with their lower extremities each in contact with the surface of the mercury in its respective instrument. The numbers therefore that stand uppermost on the numbered wheels are just those which indicate the height of the barometer and of the two thermometers, and now the same electromagnetic motor operates upon a printing apparatus which, after having deposited colour on the type, presses the slip of paper against them. This being done, the steel wires are drawn up again by the motor, which stops as soon as a certain distance from the mercury is attained, and all is ready for the next observation.

"The interval between the observations is a quarter of an hour."

Attention is then directed to the following considerations:-

"The instrument delivers the observations in a form in which they may immediately, and without further modification, be used by the meteorologist in his work.

"A very large number of very carefully made comparisons have shown that the observations registered by this method possess an accuracy equal to that which is generally attained by ocular observation.

"The zinc vessel, in which the upper ends of the thermometers are enclosed, is so air-tight that it is found possible, by means of chloride of lime and caustic potash, to keep the enclosed air always free from damp and carbonic acid, a precaution which it will be easily understood is necessary in every climate where the temperature is liable to sink below the freezing-point, but is still further necessary to protect both the mercury and the steel wires from oxidation, and thus preserve the galvanic contact.

"A meteorograph of this construction has for two years and three-quarters been in use at the Upsala Observatory, executing six observations every hour, without any perceptible alteration of the surface either of the mercury or the steel wires, that could in any way affect either the free efficiency of the instrument or its degree of accuracy, which throughout the whole time has been found to be that above named.

"As the clock which determines the time of the observations does not require winding up-the instrument itself restoring the tension of the mainspring every quarter of an hour-it continues to go as long as the driving force, i.e., the electrical current, is maintained; and, as the slip of paper applied lasts. fully three months, it is clear that that is the period for which the instrument may be left to itself. The work then requisite is little more than to take out, cut, and sew up in order the paper of observations, and replace it with another slip. We thus see that this instrument requires but very little time and labour of the person who takes charge of it.

"It is entirely for special reasons that the construction of the instrument has been limited to the registration. of thermometrical, psychrometrical (hygrometrical), and barometrical observations; for the method may be applied advantageously to observations of the course of any phenomena whatever, provided they can be indicated by an index admitting of galvanic contact. It is, therefore, applicable for all the now usual kinds of meteorological observations, and nothing prevents the same instrument executing and printing them all in one and the same table."

The following is an extract (giving one hour's instru- mental readings) of one of the printed forms referred to in the first paragraph:-

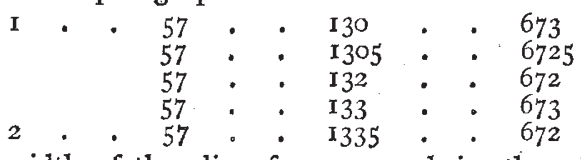

The width of the slip of paper used in these obser vations is 4.25 in.

In the Exhibition meteorograph, the timekeeper (referred to in the eighth paragraph) is merely a watch-movement of moderate size.* In the place of the ordinary minute-hand there are four, fitted on the same centre and projecting from each other at right angles in the form of a cross ; in other words, the points (one of which resembles what is technically termed a spade hour hand, and indicates the time) are $15 \mathrm{~min}$. apart. Every time one or other of the hands comes opposite the figure-III. it depresses a small steel lever which, through suitable mechanism, completes the circuit.

I am indebted to Dr. Theorell for a very courteous letter, dated from Upsala, respecting the block used in the original description, also to Messrs. Norstedt and Son, printers to the Swedish Government, for supplying me with an electrotype copy of the same through the Swedish Consulate. JOHN JAMES HALL

\section{ON SLEEP广}

PROFESSOR HUMPHRY commenced his lecture by giving a brief account of some of the changes that take place in the tissues when their function is active, and explained that during this time a slight deterioration of structure takes place, which, affecting the voluntary system, the muscles and hemispheres of the brain, causes the sense of tiring; and necessitates a period of rest for the restoration of the tissues to their former condition. In the case of the muscles this rest is provided for by periods, quickly alternating periods, of action and cessation of action. But in the case of the brain, the actions upon which conscious. ness, volition, \&c., depend cannot be thus frequently suspended. Their continuance is needed for the safety of the body during long periods, through the whole day, for instance; and longer periods are therefore required for repair. These are the periods of sleep.

He next took a cursory glance at the different parts of the nervous system, explaining that the upper regions of the brain are those which minister to consciousness and volition, the intellectual operations, \&c. He showed that the functions of these regions not only can long be suspended without interfering with the action of the lower parts of the brain, which are more immediately necessary to life; but that they are very easily suspended-slight causes, such as a jar, or a shock, or an alteration in the blood current, being sufficient to stop the action of these parts and deprive the person of consciousness. The spontaneous stopping of their action, consequent on the slight deterioration of their structure from the continuance of their functions during the day, is the proximate cause of sleep during the night; and the periodic recurrence of sleep is in accordance with the periodicity observed in several of the nutritive functions, and, indeed, witnessed in many of the other operations of nature.

Aiter observations upon the condition of the brain during sleep, the circumstances that conduce to sleep, the time that should be allotted to it, and other points, the Professor entered at some length into the subject of dreams. These he regarded not, as has been supposed by some, to be a necessary attendant on, or feature of, sleep, but rather to be the result of an abnormal condition. In the natural state we should pass from wakefulness to complete uncon-

* On the other side of the instrument to that seen in the engraving.

+ Abstract of a Lecture delivered at the Royal Institution, on Friday, February 9, by Prof. Humphry. 